\title{
Pediatric acute gastroenteritis: understanding caregivers' experiences and information needs
}

\author{
Lauren Albrecht, BEd, MEd, PhD Student**; Lisa Hartling, BSCPT, MSc, PhD**; \\ Shannon D. Scott, RN, BN, MN, PhD ${ }^{\dagger \neq}$
}

\section{ABSTRACT}

Objectives: Pediatric acute gastroenteritis (AGE) is a common condition with high health care utilization, persistent practice variation, and substantial family burden. An initial approach to resolve these issues is to understand the patient/caregiver experience of this illness. The objective of this study was to describe caregivers' experiences of pediatric AGE and identify their information needs, preferences, and priorities.

Methods: A qualitative, descriptive study was conducted. Caregivers of a child with AGE were recruited for this study in the pediatric emergency department (ED) at a tertiary hospital in a major urban centre. Individual interviews were conducted $(n=15)$, and a thematic analysis of interview transcripts was completed using a hybrid inductive/deductive approach. Results: Five major themes were identified and described: 1) caregiver management strategies; 2) reasons for going to the ED; 3) treatment and management of AGE in the ED; 4) caregivers' information needs; and 5) additional factors influencing caregivers' experiences and decision-making. A number of subthemes within each major theme were identified and described.

Conclusions: This qualitative descriptive study has identified caregiver information needs, preferences, and priorities regarding pediatric AGE. This study also identified inconsistencies in the treatment and management of pediatric AGE at home and in the ED that influence health care utilization and patient outcomes related to pediatric AGE.

\section{FRRÉSUMÉ}

Objectifs: La gastroentérite aiguë est une maladie fréquente chez les enfants, qui entraîne une grande utilisation des soins de santé, qui met en évidence des différences persistantes de pratique et qui impose un lourd fardeau à la famille. La première étape à franchir pour résoudre ces problèmes consiste à comprendre l'expérience de la maladie vécue par les enfants et les dispensateurs de soins. L'étude ici visait à décrire l'expérience de la GEA chez des enfants, vécue par des dispensateurs de soins, ainsi qu'à cerner leurs besoins d'information, leurs préférences et leurs priorités.
Méthode: II s'agit d'une étude qualitative et descriptive. Des dispensateurs de soins à des enfants souffrant d'une GEA ont été recrutés au service des urgences (SU) pédiatriques d'un hôpital de soins tertiaires situé dans un grand centre urbain. Les chercheurs ont réalisé des entrevues individuelles $(\mathrm{n}=15)$, puis ont procédé à une analyse thématique de la transcription des entrevues à l'aide d'une méthode hybride d'induction et de déduction.

Résultats: Les auteurs ont dégagé cinq grands thèmes de l'étude, puis en ont fait la description; il s'agit : 1) des stratégies de prise en charge des dispensateurs de soins; 2) des motifs de consultation au SU; 3) du traitement et de la prise en charge de la GEA au SU; 4) des besoins d'information des dispensateurs de soins; et 5) de l'existence d'autres facteurs influant sur l'expérience et la prise de décision des dispensateurs de soins. Ont aussi été dégagés de l'étude puis fait l'objet de description un certain nombre de sous-thèmes rattachés à chacun des grands thèmes. Conclusions: Cette étude qualitative et descriptive a permis de cerner les besoins d'information des dispensateurs de soins, leurs préférences et leurs priorités en ce qui concerne la GEA chez les enfants. L'étude a aussi permis de relever des différences de traitement et de prise en charge de la GEA chez les enfants, tant à domicile qu'au SU, différences susceptibles d'influer sur l'utilisation des soins de santé et les résultats cliniques observés chez les enfants souffrant d'une GEA.

Keywords: pediatric, emergency medicine, knowledge translation, information needs, health consumers

\section{INTRODUCTION}

Pediatric acute gastroenteritis (AGE) is an acute illness characterized by vomiting and diarrhea. Pediatric AGE is a common presentation in emergency departments (EDs) and remains a leading cause of global pediatric morbidity. ${ }^{1-4}$ In Canada, there are 5 million cases of pediatric AGE every year with an annual health care

From the *Department of Pediatrics and TFaculty of Nursing, University of Alberta, Edmonton, AB; and the $\neq$ Edmonton Clinic Health Academy, Edmonton, AB.

Correspondence to: Lauren Albrecht, Edmonton Clinic Health Academy, 1140587 Avenue, Edmonton, AB T6G 1C9; Email: Iauren.albrecht@ ualberta.ca 
costs of 3.7 billion dollars. $^{5}$ It is estimated that 1 in 25 children will be hospitalized for AGE by 5 years of age. ${ }^{1}$ Exacerbating the substantial health system impacts is persistent practice variation in the treatment and management of pediatric AGE. 1,6,7 Additionally, pediatric AGE affects families in a multitude of ways, including negative impacts on physical and emotional well-being of children and parents ${ }^{8}$ and frequent parental work loss. ${ }^{9}$

One proposed solution towards resolving these multifaceted issues linked to current approaches to care for pediatric AGE is to explore caregivers' perspectives of this common, acute, childhood illness. Understanding the patient/caregiver perspective may highlight misconceptions, knowledge gaps, or systemic issues contributing to the high burden of this common, acute illness. The purpose of this qualitative study was to describe caregivers' experiences of a child with pediatric AGE and to identify their information needs, preferences, and priorities.

\section{$\underline{\text { METHODS }}$}

Research ethics approval for this study was obtained from the University of Alberta Health Research Ethics Board. Participants were recruited at the Stollery Children's Hospital ED, a specialized pediatric ED in a major Canadian urban centre (Edmonton, Alberta). The inclusion criteria for study participation were 1) caregiver of a child 16 years old or younger; 2) child presenting to the Stollery Children's Hospital ED with vomiting and diarrhea; 3) caregiver fluent in English; and 4) caregiver willing to be contacted for follow-up data collection. Study exclusion criteria included 1) child with significant chronic gastrointestinal problem or inflammatory bowel disease; 2) child taking immunosuppressive therapy or known history of immunodeficiency; 3) oral or gastrointestinal surgery within the preceding 7 days; and 4) child with prior visit to ED for vomiting and diarrhea during the illness episode. Consecutive caregivers meeting the selection criteria from December 1, 2014 to January 17, 2015 during recruitment team hours (i.e., 1500-2300, 7 days a week) were approached about the study in the ED waiting room, and the project coordinator (LA) followed up with interested caregivers by telephone to schedule an interview.

Data were collected through semi-structured, qualitative interviews (Appendix A). Interviews were conducted in-person or by telephone, digitally recorded, and transcribed verbatim. This data collection strategy was used to obtain all information required, to probe participants' responses, and to give participants' freedom to respond and illustrate concepts in an open-ended fashion. ${ }^{10}$ Qualitative methods do not allow prospective determination of a sample size; however, 12-15 interviews with participants were anticipated to see patterns in experiences. ${ }^{10}$ Interview questions moved from the general to the specific with interviews later in the data collection period becoming increasingly more focused. ${ }^{11}$

Analysis was completed in NVivo $10^{12}$ and was reviewed by the research team throughout the analytic process. Thematic analysis was guided by the hybrid approach of inductive and deductive coding and theme development described by Fereday \& Muir-Cochrane (2006). ${ }^{13}$ Data saturation is not a proxy for sample adequacy or rigour in this analytic approach; the hybrid thematic analytic approached used is underpinned by Schutz's social phenomenology in which people are able to ascribe meaning to an experience or situation and then make judgments. ${ }^{14}$ To ensue rigour, this hybrid thematic analysis determined logical consistency through the initial process of deductive coding of interview transcripts using the semi-structured interview guide as a framework. This step ensured transparency in the method of formulating overarching themes. ${ }^{13,14}$ Then, smaller units of data emerged inductively and were coded for increased granularity and specificity. This step demonstrated subjective interpretation as participants' words were used to demonstrate interpretations through quotations. ${ }^{13,14}$

\section{RESULTS}

\section{Demographics}

Fifteen interviews were completed. Interviews took place ranging from 3-25 days post-ED visit with the average interview occurring 12 days post-ED visit. Interviews ranged in length from 10-23 minutes with an average interview length of 15 minutes. Demographic variables for the 15 participants are presented in Table 1. Thematic analysis of the interviews revealed five major themes: 1) caregiver management strategies; 2) reason for going to the $\mathrm{ED} ; 3$ ) treatment and management of AGE in the ED; 4) caregiver information needs; and 5) additional factors influencing caregivers' experiences and decision-making. Each of 


\begin{tabular}{|c|c|c|}
\hline Variable & Category & $\begin{array}{c}n(\%) \\
\text { [total } N=15]\end{array}$ \\
\hline \multirow[t]{2}{*}{ Caregiver's role } & Parent & $14(93)$ \\
\hline & Grandparent & $1(7)$ \\
\hline \multirow[t]{2}{*}{ Gender } & Female & $12(80)$ \\
\hline & Male & $3(20)$ \\
\hline \multirow[t]{2}{*}{ Caregiver's relationship status } & Married & $13(87)$ \\
\hline & Single & $2(13)$ \\
\hline \multirow[t]{3}{*}{ Age of caregiver } & $21-30$ years & $3(20)$ \\
\hline & $31-40$ years & $11(73)$ \\
\hline & $41-50$ years & $1(7)$ \\
\hline \multirow[t]{4}{*}{ Age of child } & Under 1 year old & $4(27)$ \\
\hline & $1-2$ years old & $8(53)$ \\
\hline & $3-5$ years old & $2(13)$ \\
\hline & Over 5 years old & $1(7)$ \\
\hline \multirow[t]{4}{*}{ Total number of children in the house } & 1 child & $6(40)$ \\
\hline & 2 children & $6(40)$ \\
\hline & 3 children & $2(13)$ \\
\hline & 4 children & $1(7)$ \\
\hline \multirow[t]{5}{*}{ Caregiver's highest level of education } & Less than high school & $2(14)$ \\
\hline & High school diploma & $2(14)$ \\
\hline & Post-secondary certificate/diploma & $3(20)$ \\
\hline & Post-secondary degree & $3(20)$ \\
\hline & Graduate degree & $5(33)$ \\
\hline \multirow[t]{5}{*}{ Average household income } & Under $\$ 25,000$ & $3(20)$ \\
\hline & $\$ 25,000-\$ 49,000$ & $2(20)$ \\
\hline & $\$ 50,000-\$ 74,999$ & $2(13)$ \\
\hline & $\$ 75,000-\$ 99,999$ & $4(27)$ \\
\hline & $\$ 100,000-\$ 149,999$ & $4(27)$ \\
\hline
\end{tabular}

these broad themes contained a number of subthemes described below. Participant quotations supporting each theme are displayed in Table 2.

\section{Theme 1: Caregiver management strategies}

As AGE signs and symptoms emerged, caregivers reported feeling scared and worried about their child with AGE and uncertain or confused about how to proceed to help their child to be well again (see Table 2). In spite of this, they engaged in a number of management strategies prior to taking the child to the ED. These strategies included putting the child to bed, giving the child a bath, changing the child's routine or environment (i.e., not going to school, sleeping on the couch), and spending time together while providing reassurance through words and touch. Caregivers also provided particular fluids, such as Pedialyte, milk, breast milk, juice, and water; some also provided specific foods, including ice cream and potatoes.
Additionally, caregivers administered medication, including Gravol, Tylenol, and Advil (see Table 2).

Prior to going into the ED, caregivers contacted health providers with questions and for advice. These providers included provincial telephone health advice service, family physicians, pediatricians, a walk-in clinic physician, and a pharmacist (see Table 2). In some cases, caregivers contacted more than one health professional. A few caregivers also sought additional advice from health providers after the ED visit, which included following up with their regular physician and in two instances, returning to the ED.

\section{Theme 2: Reason for going to the ED}

Caregivers decided to bring their child into the ED for differing reasons. Most commonly, the caregiver felt that the child's symptoms were not improving fast enough (see Table 2). Other reasons included worsening symptoms, previous experience with a similar illness 
Table 2. Participant quotes to support thematic analysis

Thematic analysis

Theme 1: caregiver management strategies

Subtheme: medication administration

Subtheme: contacting health providers

Subtheme: feeling scared/worried and uncertain/ confused

Theme 2: reason for going to the ED

Subtheme: child's symptoms not improving

Theme 3: treatment and management of AGE in the ED Subtheme: oral rehydration

Theme 4: caregiver information needs

Subtheme: caregiver questions about AGE

Subtheme: learning about effective treatments for AGE

Subtheme: conflicting information learned about the role of water for managing AGE
Participant quotes

Yeah. I mean, we tried Tylenol, but because he was barfing so much, he wasn't really keeping anything down, and then we tried a warm bath, and - but yeah, nothing really seemed to help.

(Interview 10, child age 1 year, 4 months)

I phoned the [telephone health advice] number because I wanted to know, and so did the wife actually, how long - like she couldn't do kind of - she can't keep the fluids in her, so she will get dehydrated, so we phoned, we phoned the [telephone health advice] number and got to ask about her, and that the nurse on the phone, after she went through question, she just told us since our daughter didn't - can't hold the milk for so many amount of hours now and didn't have a wet diaper and all that type of stuff to take her to the [children's hospital] to make sure she doesn't get dehydrated, so that's why we went over right to the [emergency department].

(Interview 7, child age 6 months)

[I felt] scared, confused, and powerless. (Interview 2, child age 2 years)

So we kept on doing overnight, but, like, with time he started vomiting every hour, hour and a half, but it didn't stop, so by 5 o'clock in the morning - 4:30 or 5 - then we decided, like, he's not stopping vomiting with anything so we should go to the hospital, like, it's going too much now. It was almost 12, 13 times he vomited overnight, right? So then we thought we should go, yeah.

(Interview 6, child age 2 years, 6 months)

We had to wait 20 minutes for the medication to settle and take effect, and then after the 20-minute mark, we were told that they gave us Pedialyte in a syringe, and I believe it was $10 \mathrm{ml}$. We were supposed to give it to her every 15 minutes after the 20-minute mark once that medication kicked in, so when - we were to keep hydrated to see if she was going to vomit anymore, so we gave it to her every 15 minutes, and she never vomited. She was actually taking it, and she was actually - she was gradually getting better, actually, while we were waiting in the waiting room.

(Interview 7, child age 6 months)

But yeah, like when something starts first time in home, there are so many questions in your mind, right? Why this happening, right? So what we should give him to eat, what is safe, right? So I learned a lot about that, like, some sites, like, some people say don't give milk, right? So then some say it's okay, don't give dairy products, anything like that. There was so many things, like, confusing things, but I will say just go with the simple things like Pedialyte, water. (Interview 6, child age 2 years, 6 months)

The Pedialyte popsicles are the lifesaver because they want something cold for the fever, and it's something to keep in them. Yeah. So that was - that's the biggest motherly advice I can say. If your kid is sick, make sure you have these.

(Interview 2, child age 2 years)

Yeah. Like I used to know, like, when having diarrhea, just give them fluids - I discovered later that not every kind of fluid, do not give them juice even if it is unsweetened. Just give them water.

(Interview 9, child age 1 year, 9 months)

So that was a big one that I never would've known by myself. I thought sipping 


\begin{tabular}{|c|c|}
\hline Thematic analysis & Participant quotes \\
\hline & $\begin{array}{l}\text { water was the best thing for her, and in the end when we cut the water, that's } \\
\text { what helped - that's what, like, gave her rest overnight, and I think she would've } \\
\text { been throwing up all night had I kept going. } \\
\text { (Interview 5, child age } 5 \text { years) }\end{array}$ \\
\hline Subtheme: acting sooner in future cases of AGE & $\begin{array}{l}\text { Like I think I should've taken her [to the emergency department] a day before and } \\
\text { maybe and maybe I should have because her - she already had symptoms of } \\
\text { dehydration. They might have admitted her instead of me trying to keep her home } \\
\text { as long as possible, like - I usually try to delay going to the hospitals, like, or to the } \\
\text { doctor. Try to take care at home, so that's just me. } \\
\text { (Interview 4, child age } 9 \text { years) }\end{array}$ \\
\hline Subtheme: waiting longer to act in future cases of AGE & $\begin{array}{l}\text { So in other words, I learned I probably won't go back unless I deem it to be more } \\
\text { serious and probably wait. Unfortunately, I'd probably wait for symptoms to get } \\
\text { more severe to go back. } \\
\text { (Interview } 14 \text {, child age } 5 \text { months) }\end{array}$ \\
\hline $\begin{array}{l}\text { Subtheme: use information sheet provided by hospital } \\
\text { in future cases of } A G E\end{array}$ & $\begin{array}{l}\text { I think I would go through the steps they had in the pamphlet and just make sure to } \\
\text { keep her isolated - I mean if it comes down - like keep an eye for the signs of, } \\
\text { you know, it's getting worse and severe dehydration and things like that. If it did, } \\
\text { I would still probably take her to the hospital. } \\
\text { (Interview 12, child age } 1 \text { year, } 9 \text { months) }\end{array}$ \\
\hline $\begin{array}{l}\text { Subtheme: advice for other caregivers dealing with a } \\
\text { child with AGE }\end{array}$ & $\begin{array}{l}\text { Trust your gut. } \\
\text { (Interviews 4, 11, 13, children ages } 9 \text { years, } 2 \text { years, and } 7 \text { months, respectively) }\end{array}$ \\
\hline \multicolumn{2}{|c|}{ Theme 5: additional factors influencing caregivers' experiences and decision-making } \\
\hline Subtheme: negative prior experience & $\begin{array}{l}\text { They [telephone health advice service] can't give medical advice for liability } \\
\text { reasons, so all they ever say when you call [telephone health advice service] is } \\
\text { take your kid in [to the emergency department]. } \\
\text { (Interview 14, child age } 5 \text { months) }\end{array}$ \\
\hline Subtheme: additional "life" stressors & $\begin{array}{l}\text { You knew it was bad when he stopped filling his diaper, so that's when I called the } \\
\text { doctor to see if I could get him in there, but she was completely booked up, so we } \\
\text { ended up taking him to the emergency [department]. } \\
\text { (Interview 2, child age } 2 \text { years) }\end{array}$ \\
\hline
\end{tabular}

requiring emergency care, regular physician (i.e., family doctor or pediatrician) unavailable for consult, recommendation from another health provider (i.e., telephone health advice service, walk-in clinic doctor). Additionally, one caregiver was concerned that the child's behaviour had changed and another wanted to use the latest technology in the ED for the best diagnosis, management, and treatment.

\section{Theme 3: Treatment and management of pediatric acute gastroenteritis in the $E D$}

Generally, ill children in this study were treated with oral rehydration once in the ED (see Table 2). Additionally, antiemetics (i.e., ondansetron) and/or analgesics were administered to the majority of children. A few were given extra tests, including stool samples, blood tests, and urine tests. One child was administered
IV rehydration. It is important to note that one child was provided with antibiotics for AGE on a follow-up visit with his or her regular physician post-ED discharge. During the ED visit, approximately half of caregivers indicated that they did not receive education about AGE, and of the participants who did receive education, two received verbal education only, and four received written information sheets that they could refer to after leaving the ED.

\section{Theme 4: Caregiver information needs}

Caregivers identified and described the following information needs about pediatric AGE: 1) how to alleviate AGE symptoms; 2) what to expect from a normal course of AGE; 3) how AGE is caused; 4) signs and symptoms of dehydration; 5) where to purchase helpful items (i.e., vomit bags, Pedialyte popsicles); and 4) what to tell their 
child about AGE. Caregivers expressed many questions as they reflected on and came to understand their information needs for this illness (see Table 2).

Caregivers also shared what they learned by experiencing pediatric AGE and by seeking care in the ED. They described learning about effective treatments, including over-the-counter options (i.e., Pedialyte, Pediasure, Gravol) and prescription medication (i.e., antiemetic such as ondansetron) (see Table 2). They also highlighted a better understanding of the symptoms of AGE, as well as how to recognize and deal with dehydration symptoms. There was some conflicting information learned about providing water; one caregiver learned that this was an appropriate fluid to give their child, and another reported learning that water should not be provided to children with AGE (see Table 2). One caregiver indicated that he or she had more knowledge about viruses and the seasonal nature of AGE, and another had a better understanding of nonED health providers available to consult about AGE.

Caregivers also described how new knowledge gained from this experience would impact their future actions and decisions. This largely focused on taking action sooner (i.e., go to ED earlier, provide fluids sooner, take medication earlier); however, a few noted that they would wait longer to bring their child to the ED because they felt more able to handle less severe symptoms because of this experience (see Table 2). Two caregivers stated that they would take the same course of action in the future, and another two caregivers would review the written information sheet provided by the ED to determine future actions. One caregiver indicated he or she would rather go to the ED than see the family physician because of the severity of the child's AGE in this instance, and another stated that he or she would rather see their pediatrician than visit the ED because of the wait time and lack of treatment for AGE during this experience. When asked what advice they had for other caregivers, recommendations included checking with a health provider if there was concern or doubt about the child's health and to "trust your instincts" as a parent (see Table 2) when it comes to your child's health and well-being.

\section{Theme 5: Additional factors influencing caregivers' experiences and decision-making}

In addition, caregivers described a number of factors that influenced their experience of pediatric AGE, decision-making, and actions. This included relevant past experiences, such as previously having a child with AGE, dissatisfaction with prior visits to the ED, and dissatisfaction with prior experiences of using the provincial telephone health advice service (see Table 2). Additional stressors at the time that their child was sick included multiple sick family members in the home at the same time; repeated illnesses with the same child; and the regular physician (i.e., family doctor, pediatrician) being unavailable for appointments (see Table 2). Other caregiver burdens included being the primary caregiver for the sick child and for multiple children.

\section{DISCUSSION}

The findings of this study show that caregivers employ a number of management strategies at home before seeking emergency care for pediatric AGE; however, these may be informed by popular cultural misconceptions that are not supported by best research evidence. Similarly, this study highlights inconsistencies in ED care, adding further evidence to previous research on practice variation in pediatric AGE. ${ }^{6,7}$ This study demonstrates that caregivers want to know basic information about pediatric AGE (e.g., What does AGE normally look like? What can I do to belp my child get better? What causes AGE? What should I tell my child?). However, it is clear that reasons for bringing a child to the ED and other health decisions are heavily influenced by factors reflecting "real-life" complexity, making it impossible to use one single approach to meet the needs of all caregivers and families dealing with pediatric AGE.

In this study, caregiver at home management of pediatric AGE was underpinned by common misconceptions. Caregivers reported providing their children with juice (i.e., orange juice, lemonade), milk, and ice cream, typically because it was a favourite food and they were trying to encourage their child to eat or drink. However, previous research has demonstrated that high sugar foods and fluids (e.g., juice, ice cream) may exacerbate AGE symptoms and should be avoided. ${ }^{15}$ There was also some misunderstanding regarding the role of water to combat dehydration. Oral rehydration solutions containing water and electrolytes are preferable to water alone and high sugar fluids that were provided by some caregivers in this study; however, the main consideration in determining appropriate foods and fluids to administer at home or whether to seek medical attention is the extent of dehydration. In cases of minimal or no dehydration, a regular diet 
and adequate fluids are sufficient, but these recommendations change as the severity of dehydration increases. ${ }^{16}$ Lack of understanding of appropriate fluids aligns with AGE research dating back up to 20 years. ${ }^{17,18}$ This complexity supports the need to provide specific information about what to do at home and when to seek health care to reduce non-urgent ED visits, improve patient outcomes, and reduce caregiver burden.

Inconsistencies in approaches to care were also present in ED treatment and management of pediatric AGE in this study. Approximately half of the participants $(n=7)$ indicated they did not receive education from a health care provider while in the ED. At present, it is standard practice to provide health education to parents seeking care for their children in $\mathrm{EDs}^{19}$; relevant information should be provided to all patients/ caregivers, even in cases of common, short-duration illnesses like AGE. Previous research has established that spoken medical advice is accurately remembered only $14 \%$ of the time $\mathrm{e}^{20-22}$; whereas, written information is better remembered and leads to improved treatment adherence. ${ }^{20,23}$ With no formal, written information provided to the majority of participants in this study $(\mathrm{n}=11)$, the burden is put upon caregivers to accurately remember discharge/care instructions and effectively manage their child's present and future AGE episodes.

Additionally, one health provider provided antibiotics to a child with AGE during a follow-up visit after the $\mathrm{ED}$ visit. Evidence has demonstrated that antibiotics are largely ineffective for AGE, because $75 \%$ to $90 \%$ of cases are viral ${ }^{24}$; however, in another study, $24 \%$ of pediatric emergency care and urgent care visits for AGE resulted in antibiotic prescriptions. ${ }^{25}$ This researchpractice gap is consistent with qualitative evidence highlighting pressure felt by physicians to provide antibiotics for pediatric acute infections (i.e., sore throat), even when it is known that this treatment is ineffective and inconsistent with best research evidence ${ }^{26}$ due to fear of endangering the doctor-patient relationship and a lack of understanding of patient expectations of care. ${ }^{26}$

Based on the findings of this study, it is clear that past experiences and current life circumstances affect caregiver health decision-making. For example, if caregivers are unable to consult with their regular physician, they may feel compelled to go to the ED even if they could manage their child's AGE at home. Alternately, parents with previous negative experiences with a health service (i.e., telephone health advice service or previous ED visit) may wait longer to seek care. These nuances make it difficult to determine a "one-size-fits-all" solution for patient/caregiver information provision. Understanding patients'/caregivers' experiences and connecting families to research evidence have the power to alleviate feelings of fear and uncertainty, ${ }^{9}$ ensure consistent management of child health over time and across settings, ${ }^{19}$ increase effective health decision-making, ${ }^{27}$ and reduce health system costs. ${ }^{27}$ Future research should examine the best opportunities (i.e., timing, location, mode of delivery) to provide caregivers with evidencebased information on AGE.

Caution should be used when generalizing the results of this study to other regions, populations, and child health conditions. Study participants were recruited in the ED of a tertiary care facility in an urban area in a developed country; thus findings cannot be extrapolated to caregivers that manage AGE at home without seeking emergency care, or caregivers in other types of care centres or geographic regions. Additionally, this study does not reflect the health provider perspective on the illness trajectory, the treatment and management of $\mathrm{AGE}$ in the ED, and patient information provision.

\section{CONCLUSIONS}

This study provides important information around caregivers' experiences of pediatric AGE. Qualitative approaches illuminated five major themes, including 1) caregiver management strategies; 2 ) reason for going to the ED; 3 ) treatment and management of AGE in the ED; 4) caregiver information needs; and 5) additional factors influencing caregivers' experiences and decisionmaking. Providing timely, appropriate, and engaging research-based information to caregivers about AGE may enhance their ability to communicate with health providers about their questions, concerns, and expectations for care and may also create the necessary conditions for health providers to align treatment and management with best research evidence. The challenge is to provide consistent information to caregivers that accounts for variation in the clinical presentation of AGE, potential complications of AGE and dehydration, and the experiences and needs of a diverse population of caregivers of children with AGE.

Authors' contributions: Drs. Scott and Hartling obtained research funding and designed and led the research study. Ms. Albrecht was project coordinator for this study and conducted data collection, data analysis, and manuscript writing. All 
authors provided substantive feedback and approved the manuscript prior to submission.

Acknowledgements: Authors give special thanks to Samina Ali, MD, Assistant Dean, Professionalism and Associate Professor, Departments of Pediatrics and Emergency Medicine, University of Alberta; and Nadia Dow, Research Coordinator, Department of Pediatrics, University of Alberta, for conducting paid contract services to complete initial recruitment in the ED.

Financial support: This study was funded by Canadian Institutes of Health Research Knowledge to Action Grant. Lauren Albrecht is supported by Alberta Innovates - Health Solutions Graduate Studentship and Women \& Children's Health Research Institute Graduate Studentship. Lisa Hartling is supported by a CIHR New Investigator Salary Award. Shannon Scott is a Canada Research Chair (Tier II) for Knowledge Translation in Child Health and is also supported by an Alberta Innovates Health Solutions (formerly Alberta Heritage Foundation for Medical Research) Population Health Investigator Award.

Competing interests: None declared.

\section{REFERENCES}

1. Freedman SB, Ali S, Oleszczuk M, et al. Treatment of acute gastroenteritis in children: an overview of systematic reviews of interventions commonly used in developed countries. Evid Based Child Health 2013;8:1123-37.

2. Freedman SB, Sivabalasundaram V, Bohn V, et al. The treatment of pediatrics gastroenteritis: a comparative analysis of pediatric emergency physicians' practice patterns. Acad Emerg Med 2011;18:38-45.

3. Freedman SB, Etorky M, Gorelick M, et al. Evaluation of a gastroenteritis severity score for use in outpatient settings. Pediatrics 2010;125:e1278-85.

4. Kinlin LM, Bahm A, Guttmann A, et al. A survey of emergency department resources and strategies employed in the treatment of pediatric gastroenteritis. Acad Emerg Med 2013;20(4):361-6.

5. Freedman S, Lowerison K. Alberta Provincial Pediatric Enteric Infection Team (APPETITE) seminar series. Available at: http://gotgastro.ca/training/seminar-series-2/ (accessed 2 February 2015).

6. Freedman SB, Gouin S, Bhaat $M$, et al. Prospective assessment of practice pattern variations in the treatment of pediatric gastroenteritis. Pediatrics 2011;127(2):e287-95.

7. Tieder JS, Robertson A, Garrison MM. Pediatric hospital adherence to the standard of care for acute gastroenteritis. Pediatrics 2009;124(6):e1081-6.

8. Mast TC, DeMuro-Mercon C, Kelly CM, et al. The impact of rotavirus gastroenteritis on the family. BMC Pediatr 2009;9:11.

9. Senecal M, Brisson M, Lebel MH, et al. Measuring the impact of rotavirus acute gastroenteritis episodes (MIRAGE): a prospective community-based study. Can 7 Infect Dis Med Microbiol 2008;19(6):397-404.

10. Morse J, Field P. Qualitative research methods for bealth professionals. 2nd ed. Thousand Oaks: Sage; 1995.
11. Yin R. Qualitative research from start to finish. New York: Guilford Press; 2011.

12. NVivo (for Windows) [qualitative data analysis software]. Version 10. Doncaster, Victoria, Australia: QSR International Pty Ltd.; 2012.

13. Fereday J, Muir-Cochrane E. Demonstrating rigor using thematic analysis: a hybrid approach of inductive and deductive coding and theme development. Int 7 Qual Methods 2006;5(1):80-92.

14. Schutz A. Collected papers I: the problem of social reality. Broderson A (ed.). The Hague: Martinus Nijhoff; 1973.

15. Elliot EJ. Acute gastroenteritis in children. BM7 2007;334 (7583):35-40.

16. Churgay CA, Aftab Z. Gastroenteritis in children: part II. treatment and management. Am Fam Pbysician 2012;85 (11):1066-70.

17. Li ST, Klein EJ, Tarr PI, et al. Parental management of childhood diarrhea. Clin Pediatr 2009;48(3):295-303.

18. O'Laughlin EV, Notaras E, McCullough C, et al. Home-based management of children hospitalized with acute gastroenteritis. 7 Pediatr Child Health 1995;31:189-91.

19. Taddio A, Shah V, Leung E, et al. Knowledge translation of the HELPinKIDS clinical practice guideline for managing childhood vaccination pain: usability and knowledge uptake of educational materials directed to new parents. $B M C$ Pediatr 2013;13:23.

20. Kessels R. Patients' memory for medical information. The Fournal of the Royal Society of Medicine 2003;96(5):219-22.

21. Houts PS, Bachrach R, Witmer JT, et al. Using pictographs to enhance recall of spoken medical instructions. Patient Educ Couns 1998;85:83-8.

22. Thomson AM, Cunningham SJ, Hunt NP. A comparison of information retention at an initial orthodontic consultation. Eur 7 Orthod 2001;23:169-78.

23. Blinder D, Rotenberg L, Peleg M, et al. Patient compliance to instructions after oral surgical procedures. Int 7 Oral Maxillofac Surg 2001;30:216-9.

24. Churgay CA, Aftab Z. Gastroenteritis in children: part I. diagnosis. Am Fam Physician 2012;85(11):1059-62.

25. Beatty ME, Griffin PM, Tulu A, et al. Culturing practices and antibiotic use in children with diarrhea. Pediatrics 2004;113(3):628-9.

26. Butler CC, Rollnick S, Pill R, et al. Understanding the culture of prescribing: qualitative study of general practitioners' and patients' perceptions of antiobiotics for sore throats. BM7 1998;317(7159):637-42.

27. Morrison AK, Myrvik MP, Brousseau DC, et al. The relationship between parent health literacy and pediatric emergency department utilization: a systematic review. Acad Pediatr 2013;13(5):421-9.

\section{APPENDIX A}

Semi-structured interview guide

1. Tell me about your child who was ill. How old is your child? How was your child ill? Has your child 
previously had gastroenteritis (vomiting and diarrhea)?

2. Tell me about your experience having your child experience gastroenteritis/vomiting and diarrhea.

3. How did you feel during this experience?

4. What did you do to manage your child's diarrhea and vomiting? Were there any techniques that you used?
5. What strategies were put in place by health care professionals to help your child? Did they ask you to do anything? Did they give you anything?

6. How did your child manage the experience? How did you feel about the outcome of this situation?

7. What did you learn from this experience?

8. If presented with the same situation again, would you do anything differently? 